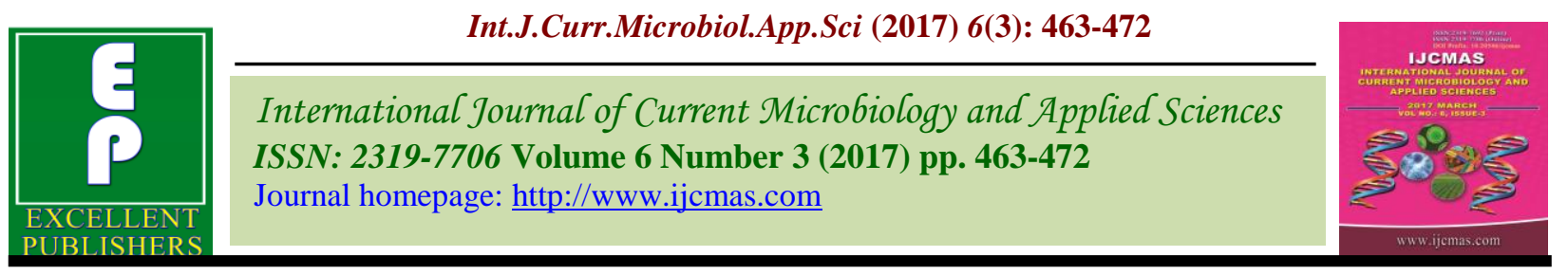

Original Research Article

https://doi.org/10.20546/ijcmas.2017.603.054

\title{
Correlation and Path Coefficient Analysis of Fruits Yield and Yield Attributes in Okra (Abelmoschus esculentus (L.) Moench)
}

\author{
G. Prasath ${ }^{1}$, K. Ravinder Reddy ${ }^{1}$ and Pidigam Saidaiah ${ }^{2}$ \\ ${ }^{1}$ Department of Horticulture, vegetable science, SKLTSHU, Rajendranagar, \\ Hyderabad-500030, Telangana, India \\ ${ }^{2}$ Department of Genetics and plant breeding, SKLTSHU, Rajendranagar, \\ Hyderabad-500030, Telangana, India \\ *Corresponding author
}

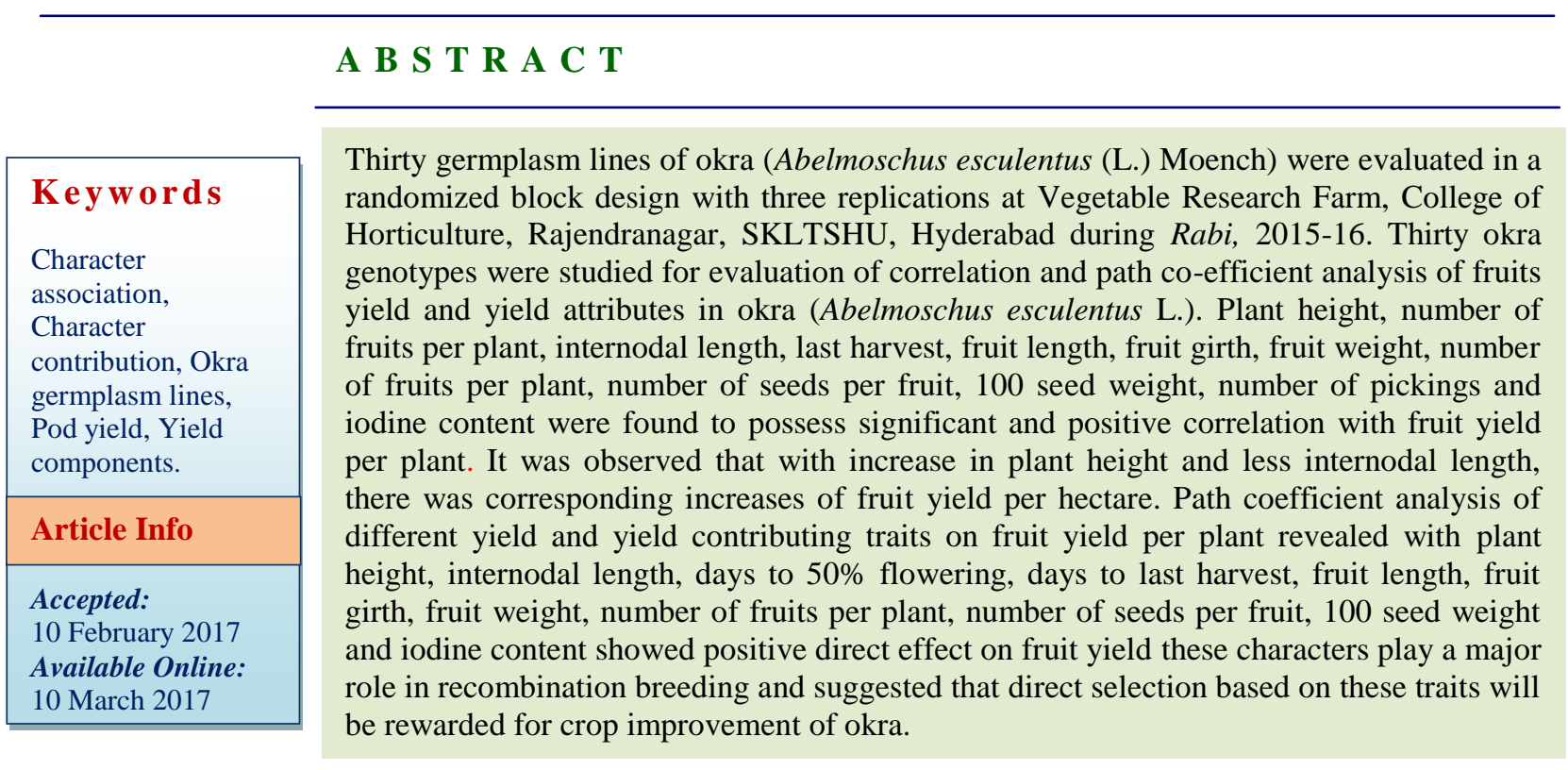

\section{Introduction}

Bhendi (Abelmoschus esculentus (L.) Moench) is popularly known as lady's finger or okra. It is the only vegetable crop of significance in the Malvaceae family. It is extensively grown in temperate, subtropical and tropical regions of the world (Kochhar, 1986). It is a specialty pod vegetable, which is very popular in India. Its fruits have high nutritive, medicinal and industrial value and export potential. Its fruits are rich in vitamins, calcium, potassium and other mineral matters
(Camciuc et al., 1981). Okra seed oil is rich in unsaturated fatty acids such as linoleic acid (Savello et al., 1980), which is essential for human nutrition. Unlike many other members of pod vegetable group, it is not strictly season-bound and hence can be gown twice a year. Being a warm season crop, it can be grown as spring-summer as well as rainy season crop in major agro-ecological zones of India. It fits well in sequential cropping systems due to its quick growing habit, 
medium duration and tolerance to drought, heat and wide variation in rainfall. Optimizing pod yield is one of the most important goals for most okra growers and, consequently, most okra breeding programs. For improving this crop through conventional breeding and selection, adequate knowledge of association that exists between yield and yield related characters is essential for the identification of selection procedure. In okra, all growth, earliness and yield associated traits are quantitative in nature. Such characters are controlled by polygenes and are much influenced by environmental fluctuations. Pod yield of okra is a complex quantitative trait, which is conditioned by the interaction of various growth and physiological processes throughout the life cycle (Adeniji and Peter, 2005). In general, plant breeders commonly select for yield components which indirectly increase yield since direct selection for yield per se may not be the most efficient method for its improvement. Indirect selection for other yield - related characters, which are closely associated with yield, will be more effective. The appropriate knowledge of such interrelationships between pod yield and its contributing components can significantly improve the efficiency of a breeding program through the use of appropriate selection indices. Correlation and path coefficient analyses are prerequisites for improvement of any crop including okra for selection of superior genotypes and improvement of any trait. In plant breeding, correlation analysis provides information about yield components and thus helps in selection of superior genotypes from diverse genetic populations. The correlation studies simply measure the associations between yield and other traits. Usefulness of the information obtained from the correlation coefficients can be enhanced by partitioning into direct and indirect effects for a set of a pair-wise cause-effect inter relationships (Kang et al., 1983). In this study, an attempt was made to study the interrelationship among characters and the direct and indirect effects of some important yield components on pod yield in germplasm lines by adopting correlation and path coefficient analysis.

\section{Materials and Methods}

Experimental material comprised 30 germplasm lines of okra. All germplasm lines were evaluated in a randomized block design with three replications at the Vegetable Research Farm, College of Horticulture, Rajendranagar, Hyderabad, during rabi, 201516. Cultural and agronomic practices were followed as per the standard recommendations and need based plant protection measures were taken up to maintain healthy crop stand. Observations were recorded on five competitive plants excluding border plants in each replication in each genotype for plant height, number of branches, internodal length, days to first flowering, days to $50 \%$ flowering, days to first harvest, days to last harvest, fruit length, fruit girth, fruit weight, number of fruits per plant, number of seeds per fruit, 100 seed weight, number of pickings, ascorbic acid content, crude fibre content, protein content, iodine content and yellow vein mosaic virus disease infestation and fruit yield per plant.

\section{Correlation coefficient analysis}

Simple correlation coefficients between yield and yield components and inter correlation among the various components were calculated using the formula suggested by Panse and Sukhatme (1967).

$$
\text { Correlation coeffient ' } r \text { ' }=\frac{\operatorname{Cov} .(X . Y)}{\sqrt{(\operatorname{Var} X)(\operatorname{Var} Y)}}
$$

Where,

$\mathrm{r}=$ Simple correlation coefficient between variable $\mathrm{X}$ and $\mathrm{Y}$ 
Cov. = Simple covariance between $X$ and

(X.Y) $\quad \mathrm{Y}$

$\mathrm{V}(\mathrm{x})=$ Variance of $\mathrm{X}$

$\mathrm{V}(\mathrm{y})=$ Variance of $\mathrm{Y}$

The significance of genotypic correlation coefficient was tested by referring to the standard table given by Snedecor (1961).

\section{Path coefficient analysis}

Path coefficient analysis was carried out as suggested by Dewey and Lu (1959) by partitioning the simple correlation coefficients into direct and indirect effects. The direct and indirect effects were ranked based on the scales of Lenka and Misra (1973) as given below

$\begin{array}{lll}\text { Negligible } & : & 0.00 \text { to } 0.09 \\ \text { Low } & : & 0.10 \text { to } 0.19 \\ \text { Moderate } & : & 0.20 \text { to } 0.29 \\ \text { High } & : & 0.30 \text { to } 0.99 \\ \text { Very high } & : & >1.00\end{array}$

\section{Results and Discussion}

\section{Correlation coefficient analysis}

Based on the simple correlation coefficients, the characters plant height, number of fruits per plant, internodal length, last harvest, fruit length, fruit girth, fruit weight, number of fruits per plant, number of seeds per fruit, 100 seed weight, number of pickings and iodine content were found to possess significant and positive association with fruit yield per plant. Such high association between fruit yield per plant, number of fruits per plant and average fruit weight was reported by Bendale et al., (2003), Jaiprakashnarayan and Mulge (2004), Somasekhar et al., (2011) and Reddy et al., (2013) in okra. The results of the present study on plant height, fruit length and fruit girth were in conformity with Niranjan and Mishra (2003), Singh et al., (2006) and Yonus et al., (2014) in okra. The results of the present study also revealed negative association of fruit yield per plant with internodal length and days to first flowering. These results corroborate the findings of Reddy et al., (2013) and Simon et al., (2013) in okra.

\section{Inter correlations among yield attributing components}

The inter correlation among component characters of yield may provide likely consequences of selection for simultaneous improvement of desirable characters. The present study revealed that plant height exhibited significant and positive correlation with number of branches, internodal length, last harvest, fruit length, fruit girth, fruit weight, number of fruits per plant, number of seeds per fruit, 100 seed weight, number of pickings, ascorbic acid and iodine content. Reddy et al., (2013) also observed positive and significant correlation of plant height with internodal length, fruit length and number of fruits per plant in okra. while it exhibited negative and significant correlation with days to first flowering, days to 50\% flowering, days to first harvest and yellow vein mosaic virus disease incidence per cent. Singh et al., (2006) also recorded negative and significant correlation of plant height with fruit girth among 19 diverse okra genotypes. Number of branches showed positive and significant correlation with internodal length, last harvest, fruit length, fruit weight, number of fruits per plant, number of seeds per fruit, 100 seed weight, number of pickings and iodine content. Internodal length showed positive and significant correlation with last harvest, fruit length, fruit weight, number of fruits per plant, number of seeds per fruit, number of pickings and iodine content (Table 1). Similar results were reported by Singh et al., (2006) and Reddy et al., (2013).

Days to first flowering showed positive and significant correlation with days to $50 \%$ 
flowering and days to last harvest. Days to $50 \%$ flowering showed significant positive correlation with days to first harvest. Days to last harvest showed positive and significant correlation with fruit girth, fruit weight, number of fruits per plant, 100 seed weight and number of pickings. Similar results were reported by Simon et al., (2013). Fruit length showed positive and significant correlation with fruit weight, number of fruits per plant, number of seeds per fruit, 100 seed weight, number of pickings and iodine content, while it exhibited negative and significant correlation with crude fibre content and protein content. Similar results were reported by Singh et al., (2006) and Reddy et al., (2013).

Fruit girth showed positive and significant correlation with fruit weight, number of fruit per plant, number pickings and ascorbic acid content. Niranjan and Mishra (2003) observed positive and significant correlation of fruit girth with plant height, number of branches, fruit length, average fruit weight, number of fruits per plant and number of seeds per fruit.

Fruit weight exhibited positive and significant correlation with number of fruits per plant, number of seeds per fruit, 100 seed weight, number of pickings and iodine content, which was accordance with the findings of Yonus et al., (2014).

Number of fruits per plant showed positive and significant correlation with number of seeds per fruit, 100 seed weight, number pickings and ascorbic acid content.

Number of seeds per fruit showed positive and significant correlation with 100 seed weight, number pickings and iodine content. Number pickings showed positive and significant correlation with ascorbic acid content and iodine content. Similar results were reported by Singh et al., (2006), Bendale et al., (2003), Reddy et al., (2013), Simon et al., (2013), Yonus et al., (2014), Niranjan and Mishra (2003).

Ascorbic acid content exhibited positive and significant correlation with crude fibre content, protein content and iodine content. Crude fibre content exhibited positive and significant correlation with protein content and iodine content. Similar results were reported by Das et al., (2012).

Further, it indicates plant height, number of branches per plant, internodal length, days to last harvest, fruit length, fruit girth, fruit weight, number of fruits per plant, number of seeds per fruit, 100 seed weight and number of pickings, ascorbic acid content and iodine content had positive and significant association with fruit yield and these characters are highly reliable components of fruit yield and could very well be utilized as yield indicators, while exercising selection.

\section{Path coefficient analysis}

The estimation of coefficients indicates only the extent and nature of association between yield and its components, but does not show the direct and indirect effects of different yield attributes on yield per se. Fruit yield is dependent on several characters which are mutually associated. These will in turn impair the true association existing between a component and fruit yield. A change in any one component is likely to disturb the whole network of cause and effect. Thus, each component has two paths of action viz., the direct influence on fruit yield, indirect effect through components which are not revealed from the correlation studies. Plant height, internodal length, days to $50 \%$ flowering, days to last harvest, fruit length, fruit girth, fruit weight, number of fruits per plant, number of seeds per fruit, 100 seed weight and iodine content showed positive direct effect on fruit yield per plant. 


\section{Int.J.Curr.Microbiol.App.Sci (2017) 6(3): 463-472}

Table.1 Phenotypic (P) and genotypic (G) correlation coefficients of yield and yield attributes in thirty genotypes of okra

\begin{tabular}{|c|c|c|c|c|c|c|c|c|c|c|c|c|c|c|c|c|c|c|c|c|c|}
\hline Characters & & 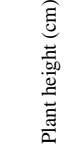 & 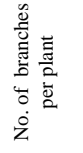 & 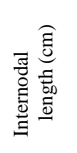 & 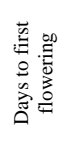 & 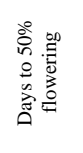 & 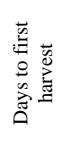 & 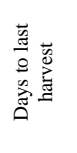 & 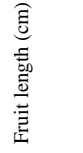 & 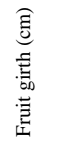 & 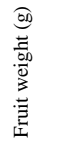 & 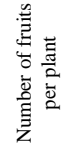 & 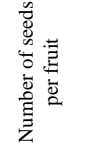 & 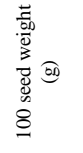 & 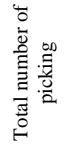 & 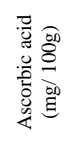 & 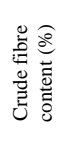 & 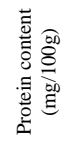 & 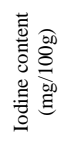 & 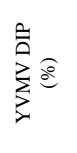 & 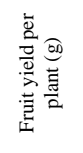 \\
\hline \multirow{2}{*}{$\begin{array}{l}\text { Plant height } \\
(\mathrm{cm})\end{array}$} & $\mathrm{P}$ & 1.0000 & $\begin{array}{l}0.7778 \\
* * * \\
\end{array}$ & $\begin{array}{l}0.3676 \\
* * * \\
\end{array}$ & $\begin{array}{l}0.5410 \\
* * *\end{array}$ & $\begin{array}{l}0.5241 \\
* *\end{array}$ & $\begin{array}{l}0.5577 \\
* * *\end{array}$ & $\begin{array}{l}0.6211 \\
* * * \\
\end{array}$ & $\begin{array}{c}0.2644 \\
*\end{array}$ & $\begin{array}{c}0.2207 \\
*\end{array}$ & $\begin{array}{c}0.6704 \\
* * * \\
\end{array}$ & $\begin{array}{c}0.7225 \\
* * * \\
\end{array}$ & $\begin{array}{c}0.4837 \\
* * \\
\end{array}$ & $\begin{array}{c}0.4734 \\
* * * \\
\end{array}$ & $\begin{array}{c}0.7082 \\
* * * \\
\end{array}$ & 0.1694 & 0.0064 & 0.0302 & $\begin{array}{c}0.3806 \\
* * * \\
\end{array}$ & $\begin{array}{c}0.5213 \\
* * * \\
\end{array}$ & $\begin{array}{l}0.7160 \\
* * * \\
\end{array}$ \\
\hline & G & 1.0000 & $\begin{array}{l}0.8662 \\
* * * \\
*\end{array}$ & $\begin{array}{l}0.4200 \\
* * \\
* *\end{array}$ & $\begin{array}{l}0.7339 \\
* * *\end{array}$ & $\begin{array}{l}0.6352 \\
* * * \\
* *\end{array}$ & $\begin{array}{l}0.7305 \\
* * *\end{array}$ & $\begin{array}{c}0.6965 \\
* * \\
\end{array}$ & $\begin{array}{c}0.2914 \\
* * * \\
\end{array}$ & $\begin{array}{c}0.2362 \\
* * * \\
\end{array}$ & $\begin{array}{c}0.7152 \\
* * * \\
\end{array}$ & $\begin{array}{c}0.7946 \\
* * * \\
\end{array}$ & $\begin{array}{c}0.5080 \\
* * \\
\end{array}$ & $\begin{array}{c}0.5069 \\
* * *\end{array}$ & $\begin{array}{c}0.7746 \\
* * * \\
\end{array}$ & 0.1695 & 0.0091 & 0.0505 & $\underset{* * *}{0.4118}$ & $\underset{* *}{0.5513}$ & $\begin{array}{l}0.7599 \\
* * * \\
\end{array}$ \\
\hline \multirow{2}{*}{$\begin{array}{l}\text { Number of } \\
\text { branches per } \\
\text { plant }\end{array}$} & P & & 1.0000 & $\begin{array}{l}0.5129 \\
* * \\
\end{array}$ & $\begin{array}{l}0.5974 \\
* *\end{array}$ & $\begin{array}{l}0.5682 \\
* * *\end{array}$ & $\begin{array}{l}0.6190 \\
* * *\end{array}$ & $\underset{* * * 6977}{0.67}$ & $\begin{array}{c}0.3641 \\
* * * \\
\end{array}$ & 0.1460 & $\begin{array}{l}0.7907 \\
* * *\end{array}$ & $\begin{array}{c}0.7750 \\
* * * \\
\end{array}$ & $\begin{array}{c}0.5664 \\
* * \\
\end{array}$ & 0.4425 & $\begin{array}{c}0.7872 \\
* * *\end{array}$ & 0.2028 & -0.0672 & 0.0091 & 0.4468 & $\underset{* *}{0.5555}$ & $\begin{array}{l}0.7977 \\
* * * \\
\end{array}$ \\
\hline & G & & 1.0000 & $\begin{array}{l}0.5632 \\
*_{* *} \\
\end{array}$ & $\begin{array}{l}0.6653 \\
* * *\end{array}$ & $\begin{array}{c}0.6159 \\
* * * \\
* *\end{array}$ & $\begin{array}{l}0.6796 \\
* * *\end{array}$ & $\begin{array}{c}0.7497 \\
* * * \\
\end{array}$ & $\begin{array}{c}0.3926 \\
* * \\
\end{array}$ & 0.1585 & $\underset{* *}{0.8349}$ & $\underset{* *}{0.8296}$ & $\underset{* *}{0.5974}$ & $\underset{* *}{0.4693}$ & $\underset{* *}{0.8445}$ & 0.2209 & -0.0713 & 0.0092 & 0.4539 & $\begin{array}{c}0.5827 \\
* * *\end{array}$ & $\begin{array}{l}0.8365 \\
* * * \\
*\end{array}$ \\
\hline \multirow{2}{*}{$\begin{array}{l}\text { Intermodal } \\
\text { length }(\mathrm{cm})\end{array}$} & P & & & 1.0000 & $\begin{array}{l}0.3694 \\
* * *\end{array}$ & $\begin{array}{l}0.3701 \\
* * \\
* *\end{array}$ & $\begin{array}{l}0.3681 \\
* * * \\
* *\end{array}$ & $\begin{array}{l}0.2732 \\
* * *\end{array}$ & $\begin{array}{l}0.5426 \\
* * *\end{array}$ & -0.1619 & $\begin{array}{l}0.5258 \\
* * * \\
*\end{array}$ & $\begin{array}{l}0.4505 \\
* *\end{array}$ & $\begin{array}{l}0.4989 \\
* *\end{array}$ & 0.1162 & $\begin{array}{l}0.4994 \\
* * *\end{array}$ & 0.0207 & $\begin{array}{l}0.2779 \\
* * *\end{array}$ & $\begin{array}{l}0.2585 \\
* \\
*\end{array}$ & $\begin{array}{l}0.3837 \\
* * * \\
* *\end{array}$ & $\begin{array}{l}0.3295 \\
* *\end{array}$ & $\begin{array}{l}0.5007 \\
* * \\
* *\end{array}$ \\
\hline & G & & & 1.0000 & $\begin{array}{l}0.4401 \\
* *\end{array}$ & $\begin{array}{l}0.4308 \\
* * \\
* *\end{array}$ & $\begin{array}{l}0.4355 \\
* * * \\
*\end{array}$ & $\begin{array}{l}0.3326 \\
* * \\
\end{array}$ & $\begin{array}{l}0.5902 \\
* * * \\
*\end{array}$ & -0.1865 & $\begin{array}{l}0.5644 \\
* * * \\
*\end{array}$ & $\begin{array}{l}0.4903 \\
* *\end{array}$ & $\begin{array}{l}0.5429 \\
* *\end{array}$ & 0.1197 & $\begin{array}{l}0.5560 \\
* * * \\
*\end{array}$ & 0.0303 & $\begin{array}{l}0.2910 \\
* * *\end{array}$ & $\begin{array}{l}0.2822 \\
*\end{array}$ & $\begin{array}{l}0.4152 \\
* * * \\
\end{array}$ & $\begin{array}{l}0.3490 \\
* * *\end{array}$ & $\begin{array}{l}0.5332 \\
* * \\
* * *\end{array}$ \\
\hline \multirow{2}{*}{$\begin{array}{l}\text { Days to first } \\
\text { flowering }\end{array}$} & P & & & & 1.0000 & $\begin{array}{l}0.9362 \\
* *\end{array}$ & $\begin{array}{l}0.9763 \\
* * *\end{array}$ & $\begin{array}{l}- \\
0.3380 \\
*_{* *}\end{array}$ & $\begin{array}{l}- \\
0.2142 \\
*\end{array}$ & $\begin{array}{l}- \\
0.3154 \\
* *\end{array}$ & $\begin{array}{l}0.5843 \\
* * \\
* *\end{array}$ & $\begin{array}{l}- \\
0.4804 \\
* *\end{array}$ & $\begin{array}{l}0.4790 \\
* *\end{array}$ & $\begin{array}{l}- \\
0.3333 \\
* *\end{array}$ & $\begin{array}{l}0.4671 \\
* * \\
* *\end{array}$ & -0.0775 & -0.0195 & -0.0526 & $\begin{array}{l}0.2970 \\
* * \\
* *\end{array}$ & $\begin{array}{l}0.5939 \\
* *\end{array}$ & -0.4971 \\
\hline & G & & & & 1.0000 & $\begin{array}{l}0.9920 \\
* * \\
\end{array}$ & $\begin{array}{l}0.9811 \\
* * * \\
\end{array}$ & $\begin{array}{l}0.4037 \\
* * * \\
* *\end{array}$ & $\begin{array}{l}0.2495 \\
* \\
*\end{array}$ & $\begin{array}{l}0.3634 \\
* * * \\
* *\end{array}$ & $\begin{array}{l}0.6699 \\
* * * \\
* *\end{array}$ & $\begin{array}{l}0.6228 \\
* * * \\
*\end{array}$ & $\begin{array}{l}0.5551 \\
* * \\
\end{array}$ & $\begin{array}{l}0.3903 \\
* * * \\
*\end{array}$ & $\begin{array}{l}0.5876 \\
* * * \\
*\end{array}$ & -0.0954 & -0.0232 & -0.0673 & $\begin{array}{l}0.3436 \\
* * * \\
*\end{array}$ & $\begin{array}{l}0.6915 \\
* *\end{array}$ & -0.5725 \\
\hline \multirow{2}{*}{$\begin{array}{l}\text { Days to } 50 \% \\
\text { flowering }\end{array}$} & $\mathrm{P}$ & & & & & 1.0000 & $\begin{array}{l}0.9470 \\
* * * \\
\end{array}$ & $\begin{array}{l}0.2964 \\
* * * \\
* *\end{array}$ & $\begin{array}{l}0.2359 \\
* \\
\end{array}$ & -.03080 & $\begin{array}{l}0.5663 \\
* * * \\
* *\end{array}$ & $\begin{array}{l}0.5678 \\
* * * \\
* * *\end{array}$ & $\begin{array}{l}0.4906 \\
* * \\
\end{array}$ & $\begin{array}{l}0.3204 \\
* * * \\
* *\end{array}$ & $\begin{array}{l}0.4701 \\
* * \\
* *\end{array}$ & -0.1886 & -0.0670 & -0.1135 & $\begin{array}{l}0.3411 \\
* * \\
* *\end{array}$ & $\begin{array}{l}0.6476 \\
* *\end{array}$ & -0.5028 \\
\hline & G & & & & & 1.0000 & $\begin{array}{l}0.9930 \\
* * * \\
*\end{array}$ & $\begin{array}{l}0.3190 \\
* *\end{array}$ & $\begin{array}{l}0.2451 \\
*\end{array}$ & -0.3342 & $\begin{array}{l}0.5989 \\
* * * \\
* *\end{array}$ & $\begin{array}{l}0.6425 \\
* *\end{array}$ & $\begin{array}{l}0.5239 \\
* *\end{array}$ & $\begin{array}{l}0.3422 \\
* * * \\
\end{array}$ & $\begin{array}{l}0.5382 \\
* * *\end{array}$ & -0.2095 & -0.0689 & -0.1228 & $\begin{array}{l}0.3619 \\
* * * \\
* *\end{array}$ & $\begin{array}{l}0.6925 \\
* *\end{array}$ & -0.5357 \\
\hline \multirow{2}{*}{$\begin{array}{c}\text { Days to first } \\
\text { harvest }\end{array}$} & $\mathrm{P}$ & & & & & & 1.0000 & $\begin{array}{l}- \\
0.3380 \\
*_{* *}\end{array}$ & $\begin{array}{l}- \\
0.2107 \\
*\end{array}$ & $\begin{array}{l}0.2927 \\
* * \\
* *\end{array}$ & $\begin{array}{l}0.5689 \\
* * \\
* *\end{array}$ & $\begin{array}{l}0.5406 \\
* * \\
* *\end{array}$ & $\begin{array}{l}0.4873 \\
* * *\end{array}$ & $\begin{array}{l}0.3827 \\
* * \\
* *\end{array}$ & $\begin{array}{l}0.4697 \\
* * *\end{array}$ & -0.1359 & -0.1033 & -0.1342 & $\begin{array}{l}0.3240 \\
* * *\end{array}$ & $\begin{array}{l}0.6292 \\
* *\end{array}$ & -0.5020 \\
\hline & G & & & & & & 1.0000 & $\begin{array}{l}0.3994 \\
* * *\end{array}$ & $\begin{array}{l}0.2351 \\
* *\end{array}$ & $\begin{array}{l}0.3335 \\
* * *\end{array}$ & $\begin{array}{l}0.6384 \\
* * *\end{array}$ & $\begin{array}{l}0.6750 \\
* * *\end{array}$ & $\begin{array}{c}0.5489 \\
* * \\
\end{array}$ & $\begin{array}{l}0.4356 \\
* * *\end{array}$ & $\begin{array}{l}0.5730 \\
* * *\end{array}$ & -0.1590 & -0.1180 & -0.1593 & -0.3642 & $\begin{array}{l}0.7117 \\
* * *\end{array}$ & -0.5618 \\
\hline \multirow{2}{*}{$\begin{array}{c}\text { Days to last } \\
\text { harvest }\end{array}$} & P & & & & & & & 1.0000 & 0.1398 & $\begin{array}{l}0.3151 \\
* * \\
\end{array}$ & $\begin{array}{l}0.7172 \\
* * * \\
* *\end{array}$ & $\begin{array}{l}0.5964 \\
* *\end{array}$ & 0.2010 & $\begin{array}{l}0.2318 \\
*\end{array}$ & $\begin{array}{l}0.7579 \\
* * * \\
\end{array}$ & 0.0747 & -0.0562 & 0.0795 & 0.1719 & $\begin{array}{l}0.3798 \\
* * *\end{array}$ & $\begin{array}{l}0.7384 \\
*\end{array}$ \\
\hline & G & & & & & & & 1.0000 & 0.1512 & $\begin{array}{l}0.3435 \\
* * * \\
\end{array}$ & $\begin{array}{l}0.7690 \\
* * * \\
\end{array}$ & $\begin{array}{l}0.6451 \\
*_{* *}\end{array}$ & 0.2155 & $\begin{array}{l}0.2471 \\
*\end{array}$ & $\begin{array}{l}0.8404 \\
* * * \\
\end{array}$ & 0.0791 & -0.0585 & 0.0898 & 0.1828 & $\begin{array}{l}0.4026 \\
* *\end{array}$ & $\begin{array}{l}0.7860 \\
*\end{array}$ \\
\hline \multirow{2}{*}{$\begin{array}{l}\text { Fruit length } \\
(\mathrm{cm})\end{array}$} & P & & & & & & & & 1.0000 & -0.0838 & $\begin{array}{l}0.3987 \\
* *\end{array}$ & $\underset{* *}{0.3725}$ & $\begin{array}{l}0.5435 \\
* * *\end{array}$ & $\begin{array}{l}0.3545 \\
* *\end{array}$ & $\begin{array}{l}0.3523 \\
* * *\end{array}$ & 0.0696 & $\begin{array}{l}0.2514 \\
*\end{array}$ & $\begin{array}{l}0.3241 \\
* * * \\
\end{array}$ & $\begin{array}{l}0.4972 \\
* * * \\
* *\end{array}$ & $\begin{array}{l}0.3311 \\
* * \\
\end{array}$ & $\begin{array}{l}0.4670 \\
* * *\end{array}$ \\
\hline & G & & & & & & & & 1.0000 & -0.0868 & $\begin{array}{l}0.4039 \\
* *\end{array}$ & $\begin{array}{l}0.3963 \\
* *\end{array}$ & $\begin{array}{l}0.5592 \\
* *\end{array}$ & $\begin{array}{l}0.3614 \\
* * \\
\end{array}$ & $\begin{array}{l}0.3730 \\
* *\end{array}$ & 0.0697 & $\begin{array}{l}0.2551 \\
* \\
*\end{array}$ & $\begin{array}{l}0.3298 \\
* * *\end{array}$ & $\begin{array}{l}0.5098 \\
* * \\
* *\end{array}$ & $\begin{array}{l}0.3359 \\
* *\end{array}$ & $\begin{array}{l}0.4753 \\
* * \\
\end{array}$ \\
\hline \multirow{2}{*}{$\begin{array}{l}\text { Fruit girth } \\
\quad(\mathrm{cm})\end{array}$} & $\mathrm{P}$ & & & & & & & & & 1.0000 & $\begin{array}{l}0.3604 \\
* * *\end{array}$ & $\begin{array}{l}0.2727 \\
* *\end{array}$ & -0.2118 & 0.1003 & $\begin{array}{l}0.3268 \\
* * *\end{array}$ & $\begin{array}{l}0.2212 \\
*\end{array}$ & 0.1362 & 0.0673 & -0.0314 & $\begin{array}{l}0.4429 \\
* * \\
\end{array}$ & $\begin{array}{l}0.3452 \\
* * *\end{array}$ \\
\hline & G & & & & & & & & & 1.0000 & $\begin{array}{l}0.3700 \\
* * * \\
*\end{array}$ & $\begin{array}{l}0.2921 \\
* * * \\
\end{array}$ & -0.2150 & 0.1018 & $\begin{array}{l}0.3469 \\
* * * \\
{ }_{*}^{*}\end{array}$ & $\begin{array}{l}0.2268 \\
* \\
\end{array}$ & 0.1381 & 0.0683 & -0.0263 & $\begin{array}{l}0.4487 \\
* *\end{array}$ & $\begin{array}{l}0.3513 \\
* * * \\
*\end{array}$ \\
\hline
\end{tabular}


Int.J.Curr.Microbiol.App.Sci (2017) 6(3): 463-472

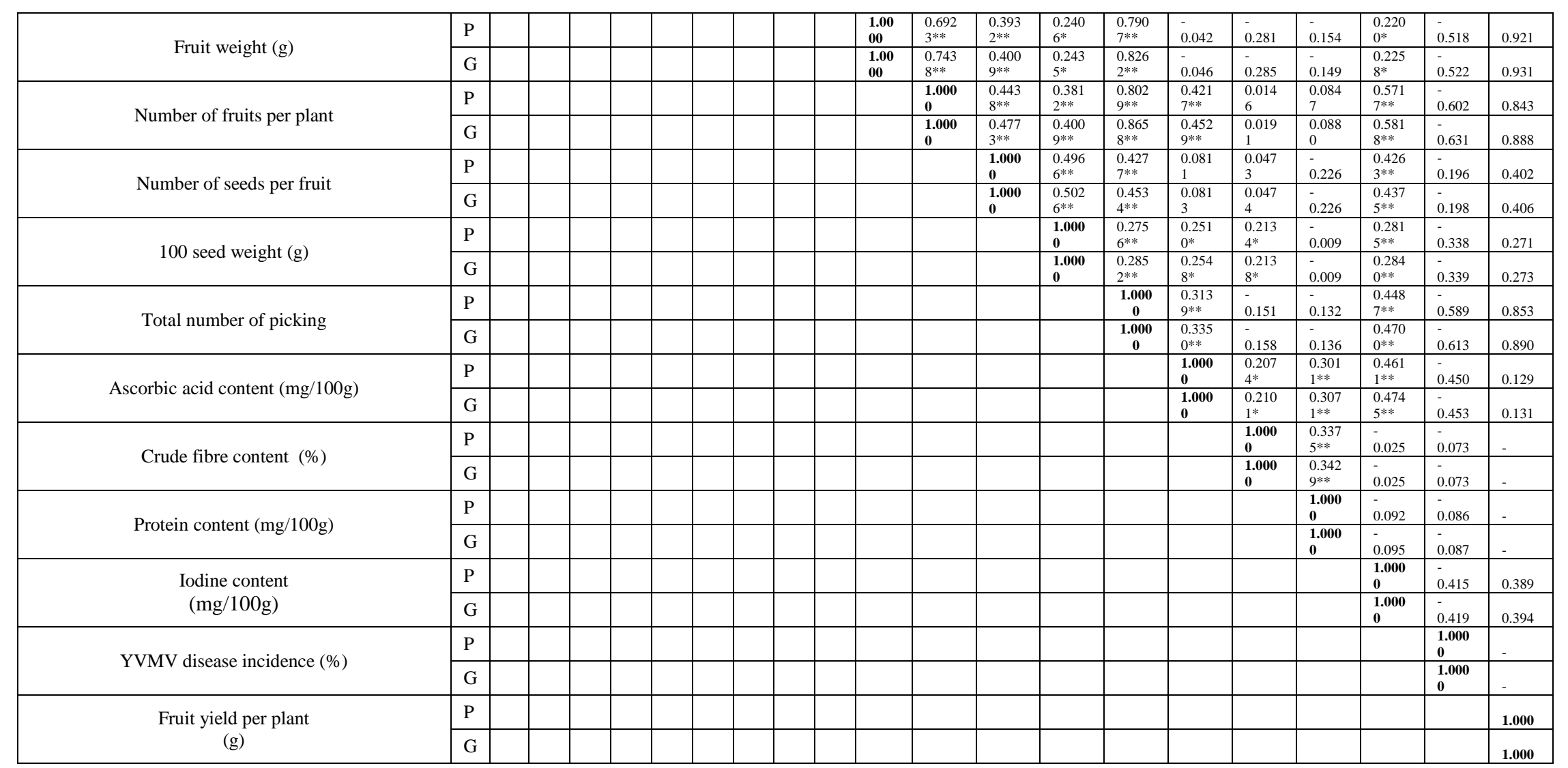

*Significant at 5 per cent level; ** Significant at 1 per cent level 
Table.2 Phenotypic (P) and genotypic $(\mathrm{G})$ path coefficient analysis indicating direct and indirect effects of component characters on fruit yield in thirty genotypes of okra

\begin{tabular}{|c|c|c|c|c|c|c|c|c|c|c|c|c|c|c|c|c|c|c|c|c|c|}
\hline $\begin{array}{c}\text { Character } \\
\text { s }\end{array}$ & & 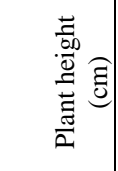 & 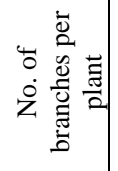 & 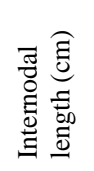 & 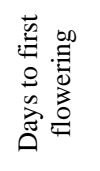 & 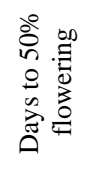 & 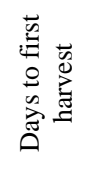 & 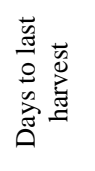 & 苟 & 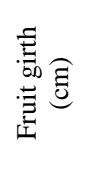 & 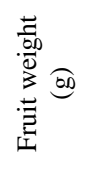 & 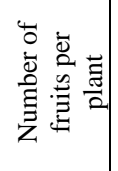 & 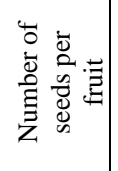 & 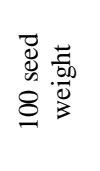 & 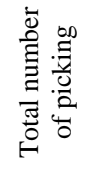 & 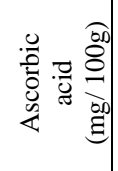 & 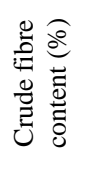 & 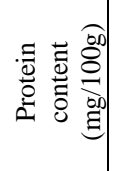 & 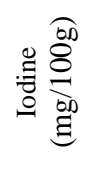 & $\sum_{i}^{\infty} \hat{0}$ & 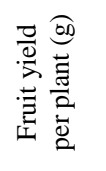 \\
\hline \multirow{2}{*}{$\begin{array}{l}\text { Plant } \\
\text { height } \\
(\mathrm{cm})\end{array}$} & $\mathbf{P}$ & $\underline{0.0304}$ & 0.0236 & 0.0112 & -0.0164 & -0.0159 & -0.0169 & 0.0189 & 0.0080 & 0.0067 & 0.0204 & 0.0219 & 0.0147 & 0.0144 & 0.0215 & 0.0051 & 0.0002 & 0.0009 & 0.0116 & -0.0158 & $0.7160^{* *}$ \\
\hline & $\mathbf{G}$ & $\underline{-0.2438}$ & -0.2112 & -0.1024 & 0.1790 & 0.1549 & 0.1781 & -0.1698 & $\begin{array}{l}- \\
.0 .0711\end{array}$ & -0.0576 & -0.1744 & -0.1938 & -0.1239 & -0.1236 & -0.1889 & -0.0412 & -0.0022 & -0.0123 & -0.1004 & 0.1344 & $0.7599^{* * *}$ \\
\hline \multirow{2}{*}{$\begin{array}{l}\text { Number } \\
\text { of } \\
\text { branches } \\
\text { per plant }\end{array}$} & $\mathbf{P}$ & -0.0790 & $\underline{-0.1060}$ & -0.0521 & 0.0607 & 0.0577 & 0.0629 & -0.0709 & -0.0370 & -0.0148 & -0.0804 & -0.0788 & -0.0576 & -0.0450 & -0.0800 & -0.0206 & 0.0068 & -0.0009 & -0.0454 & 0.0565 & $0.7977^{* *}$ \\
\hline & $\mathbf{G}$ & -0.0756 & $\underline{-0.0873}$ & -0.0492 & 0.0581 & 0.0538 & 0.0593 & -0.0654 & -0.0343 & -0.0138 & -0.0729 & -0.0724 & -0.0521 & -0.0410 & -0.0737 & -0.0193 & 0.0062 & -0.0005 & -0.0396 & 0.0509 & $0.8365^{* *}$ \\
\hline \multirow{2}{*}{$\begin{array}{l}\text { Intermod } \\
\text { al length } \\
\quad(\mathrm{cm})\end{array}$} & $\mathbf{P}$ & -0.0269 & -0.0375 & $\underline{-0.0731}$ & 0.0270 & 0.0271 & 0.0269 & -0.0200 & -0.0397 & 0.0118 & -0.0384 & -0.0329 & -0.0365 & -0.0085 & -0.0365 & -0.0015 & 0.0203 & 0.0189 & -0.0280 & 0.0241 & $0.5007^{* * *}$ \\
\hline & $\mathbf{G}$ & 0.1274 & 0.1709 & $\underline{0.3034}$ & -0.1335 & -0.1307 & -0.1321 & 0.1009 & 0.1791 & -0.0566 & 0.1713 & 0.1487 & 0.1647 & 0.0363 & 0.1687 & 0.0092 & -0.0883 & -0.0856 & 0.1260 & -0.1059 & $0.5332 * *$ \\
\hline \multirow{2}{*}{$\begin{array}{c}\text { Days to } \\
\text { first } \\
\text { flowerin } \\
\mathrm{g} \\
\end{array}$} & $\mathbf{P}$ & 0.0796 & 0.0879 & 0.0543 & $\underline{-0.1471}$ & -0.1377 & -0.1436 & 0.0497 & 0.0315 & 0.0464 & 0.0860 & 0.0707 & 0.0705 & 0.0490 & 0.0687 & 0.0114 & 0.0029 & 0.0077 & 0.0437 & -0.0874 & -0.4971 \\
\hline & $\mathbf{G}$ & 1.9712 & 1.7869 & 1.1821 & $\underline{-2.6858}$ & -2.6644 & -2.6351 & 1.0843 & 0.6700 & 0.9761 & 1.7992 & 1.6727 & 1.4908 & 1.0484 & 1.5782 & 0.2563 & 0.0623 & 0.1808 & 0.9228 & -1.8573 & -0.5725 \\
\hline \multirow{2}{*}{$\begin{array}{c}\text { Days to } \\
50 \% \\
\text { flowerin } \\
\mathrm{g} \\
\end{array}$} & $\mathbf{P}$ & -0.1471 & -0.1595 & -0.1039 & 0.2628 & $\underline{0.2807}$ & 0.2658 & -0.0832 & -0.0662 & -0.0865 & -0.1590 & -0.1594 & -0.1377 & -0.0899 & -0.1320 & -0.0529 & -0.0188 & -0.0318 & -0.0957 & 0.1818 & -0.5028 \\
\hline & $\mathbf{G}$ & 0.0801 & 0.0777 & 0.0543 & -0.1251 & $\underline{-0.1261}$ & -0.1252 & 0.0402 & 0.0309 & 0.0421 & 0.0755 & 0.0810 & 0.0661 & 0.0431 & 0.0679 & 0.0264 & 0.0087 & 0.0155 & 0.0456 & -0.0873 & -0.5357 \\
\hline \multirow{2}{*}{$\begin{array}{c}\text { Days to } \\
\text { first } \\
\text { harvest }\end{array}$} & $\mathbf{P}$ & 0.0048 & 0.0053 & 0.0032 & -0.0084 & -0.0081 & $\underline{-0.0086}$ & 0.0029 & 0.0018 & 0.0025 & 0.0049 & 0.0046 & 0.0042 & 0.0033 & 0.0040 & 0.0012 & 0.0009 & 0.0012 & 0.0028 & -0.0054 & -0.5020 \\
\hline & $\mathbf{G}$ & -2.5289 & -2.3526 & -1.5076 & 3.3965 & 3.4375 & $\underline{3.4619}$ & -1.3825 & -0.8137 & -1.1544 & -2.2101 & -2.3368 & -1.9004 & -1.5080 & -1.9835 & -0.5505 & -0.4086 & -0.5514 & -1.2609 & 2.4640 & -0.5618 \\
\hline \multirow{2}{*}{$\begin{array}{c}\text { Days to } \\
\text { last } \\
\text { harvest }\end{array}$} & $\mathbf{P}$ & 0.0627 & 0.0704 & 0.0276 & -0.0341 & -0.0299 & -0.0341 & $\underline{0.1009}$ & 0.0141 & 0.0318 & 0.0724 & 0.0602 & 0.0203 & 0.0234 & 0.0765 & 0.0075 & -0.0057 & 0.0080 & 0.0173 & -0.0383 & $0.7384 *$ \\
\hline & $\mathbf{G}$ & 1.0423 & 1.1219 & 0.4977 & -0.6042 & -0.4774 & -0.5976 & $\underline{1.4965}$ & 0.2263 & 0.5140 & 1.1508 & 0.9654 & 0.3225 & 0.3697 & 1.2576 & 0.1174 & -0.0876 & 0.1344 & 0.2436 & -0.6024 & $0.7860^{*}$ \\
\hline \multirow{2}{*}{$\begin{array}{l}\text { Fruit } \\
\text { length } \\
(\mathrm{cm})\end{array}$} & $\mathbf{P}$ & 0.0258 & 0.0356 & 0.0530 & -0.0209 & -0.0231 & -0.0206 & 0.0137 & $\underline{0.0978}$ & -0.0082 & 0.0390 & 0.0364 & 0.0531 & 0.0347 & 0.0344 & 0.0068 & -0.0246 & -0.0317 & 0.0486 & -0.0324 & $0.4670 * *$ \\
\hline & $\mathbf{G}$ & -0.0854 & -0.1150 & -0.1729 & 0.0731 & 0.0718 & 0.0689 & -0.0443 & $\underline{-0.2929}$ & 0.0254 & -0.1183 & -0.1161 & -0.1638 & -0.1059 & -0.1093 & -0.0203 & 0.0747 & 0.0966 & -0.1494 & 0.0984 & $0.4753 * *$ \\
\hline \multirow{2}{*}{$\begin{array}{l}\text { Fruit } \\
\text { girth } \\
(\mathrm{cm})\end{array}$} & $\mathbf{P}$ & 0.0039 & 0.0026 & -0.0029 & -0.0056 & -0.0054 & -0.0052 & 0.0056 & -0.0015 & $\underline{0.0177}$ & 0.0064 & 0.0048 & -0.0037 & 0.0018 & 0.0058 & 0.0039 & 0.0024 & 0.0012 & -0.0006 & -0.0078 & $0.3452 * *$ \\
\hline & $\mathbf{G}$ & 0.0568 & 0.0381 & -0.0449 & -0.0875 & -0.0804 & -0.0803 & 0.0827 & -0.0209 & $\underline{0.2407}$ & 0.0891 & 0.0703 & -0.0518 & 0.0245 & 0.0835 & 0.0546 & 0.0332 & 0.0164 & -0.0063 & -0.1080 & $0.3513 * *$ \\
\hline
\end{tabular}


Int.J.Curr.Microbiol.App.Sci (2017) 6(3): 463-472

\begin{tabular}{|c|c|c|c|c|c|c|c|c|c|c|c|c|c|c|c|c|c|c|c|c|c|}
\hline \multirow{2}{*}{$\begin{array}{c}\text { Fruit } \\
\text { weight }(\mathrm{g})\end{array}$} & $P$ & 0.3876 & 0.4572 & 0.3040 & -0.3378 & -0.3274 & -0.3289 & 0.4147 & 0.2305 & 0.2084 & $\underline{0.5782}$ & 0.4003 & 0.2273 & 0.1391 & 0.4572 & -0.0247 & -0.1629 & -0.0891 & 0.1272 & -0.2997 & $0.9214^{* *}$ \\
\hline & G & -0.1593 & -0.1860 & -0.1257 & 0.1492 & 0.1334 & 0.1422 & -0.1713 & -0.0900 & -0.0824 & $\underline{-0.2227}$ & -0.1657 & -0.0893 & -0.0542 & -0.1840 & 0.0103 & 0.0635 & 0.0333 & -0.0503 & 0.1164 & $0.9317^{* *}$ \\
\hline \multirow{2}{*}{$\begin{array}{l}\text { Number of } \\
\text { fruits per } \\
\text { plant }\end{array}$} & $\mathrm{P}$ & 0.3525 & 0.3781 & 0.2198 & -0.2344 & -0.2770 & -0.2638 & 0.2910 & 0.1817 & 0.1330 & 0.3377 & $\underline{0.4879}$ & 0.2165 & 0.1860 & 0.3917 & 0.2057 & 0.0071 & 0.0413 & 0.2789 & -0.2938 & $0.8430 * *$ \\
\hline & G & 1.9529 & 2.0389 & 1.2048 & -1.5306 & -1.5790 & -.6589 & 1.5855 & 0.9740 & 0.7179 & 1.8280 & $\underline{2.4576}$ & 1.1730 & 0.9853 & 2.1278 & 1.1131 & 0.0470 & 0.1672 & 1.4299 & -1.5525 & $0.8883^{* *}$ \\
\hline \multirow{2}{*}{$\begin{array}{c}\text { Number of } \\
\text { seeds per fruit }\end{array}$} & $P$ & 0.0295 & 0.0346 & 0.0305 & -0.0292 & -0.0300 & -0.0298 & 0.0123 & 0.0332 & -0.0129 & 0.0240 & 0.0271 & $\underline{0.0611}$ & 0.0303 & 0.0261 & 0.0050 & 0.0029 & -0.0138 & 0.0260 & -0.0120 & $0.4027^{* *}$ \\
\hline & G & 0.3368 & 0.3960 & 0.3599 & -0.3680 & -0.3473 & -0.3639 & 0.1429 & 0.3707 & -0.1426 & 0.2658 & 0.3164 & $\underline{0.6629}$ & 0.3332 & 0.3005 & 0.0536 & 0.0311 & -0.1502 & 0.2900 & -0.1319 & $0.4067^{* *}$ \\
\hline \multirow{2}{*}{$\begin{array}{c}100 \text { seed } \\
\text { weight }(\mathrm{g})\end{array}$} & $\mathrm{P}$ & -0.0343 & -0.0320 & -0.0084 & 0.0241 & 0.0232 & 0.0277 & -0.0168 & -0.0257 & -0.0073 & -0.0174 & -0.0276 & -0.0360 & $\underline{-0.0724}$ & -0.0200 & -0.0182 & -0.0155 & 0.0007 & -0.0204 & 0.0245 & $0.2715^{* *}$ \\
\hline & G & -0.0656 & -0.0607 & -0.0155 & 0.0505 & 0.0443 & 0.0563 & -0.0320 & -0.0467 & -0.0132 & -0.0315 & -0.0519 & -0.0650 & $\underline{-0.1294}$ & -0.0369 & -0.0330 & -0.0277 & 0.0012 & -0.0367 & 0.0469 & $0.2734 * *$ \\
\hline \multirow{2}{*}{$\begin{array}{l}\text { Total number } \\
\text { of picking }\end{array}$} & $P$ & -0.0123 & -0.0137 & -0.0087 & 0.0081 & 0.0082 & 0.0082 & -0.0132 & -0.0061 & -0.0057 & -0.0138 & -0.0140 & -0.0075 & -0.0048 & $\underline{-0.0174}$ & -0.0055 & 0.0026 & 0.0023 & -0.0078 & 0.0103 & $0.8536^{* *}$ \\
\hline & G & -1.9860 & -2.1650 & -1.4250 & 1.5065 & 1.3799 & 1.4689 & -2.1546 & -0.9563 & -0.8891 & -2.1182 & -2.2198 & -1.1623 & -0.7311 & $\underline{-2.5638}$ & -0.8590 & 0.4051 & 0.3490 & -1.2051 & 1.5722 & $0.8909^{* *}$ \\
\hline \multirow{2}{*}{$\begin{array}{c}\text { Ascorbic acid } \\
\text { content } \\
(\mathrm{mg} / 100 \mathrm{~g})\end{array}$} & $P$ & -0.0030 & -0.0036 & -0.0004 & 0.0014 & 0.0034 & 0.0024 & -0.0013 & -0.0013 & -0.0040 & 0.0008 & -0.0076 & -0.0015 & -0.0045 & -0.0056 & $\underline{-0.0180}$ & -0.0037 & -0.0054 & -0.0083 & -0.0081 & $0.1290^{*}$ \\
\hline & G & -0.0258 & -0.0337 & -0.0046 & 0.0145 & 0.0319 & 0.0242 & -0.0121 & -0.0106 & -0.0346 & 0.0070 & -0.0690 & -0.0123 & -0.0388 & -0.0511 & $\underline{-0.1524}$ & -0.0320 & -0.0468 & -0.0723 & 0.0692 & $0.1312^{*}$ \\
\hline \multirow{2}{*}{$\begin{array}{l}\text { Crude fibre } \\
\text { content }(\%)\end{array}$} & $\mathrm{P}$ & -0.0004 & 0.0043 & 0.0176 & 0.0012 & 0.0042 & 0.0066 & 0.0036 & 0.0159 & -0.0086 & 0.0179 & -0.0009 & -0.0030 & -0.0135 & 0.0096 & -0.0132 & $\underline{-0.0634}$ & -0.0214 & 0.0016 & 0.0047 & -0.2440 \\
\hline & G & -0.0028 & 0.0222 & 0.0904 & 0.0072 & 0.0214 & 0.0367 & 0.0182 & 0.0793 & -0.0429 & 0.0886 & -0.0059 & -0.0146 & -0.0664 & 0.0491 & -0.0653 & $\underline{-0.3107}$ & -0.1066 & 0.0078 & 0.0229 & -0.2447 \\
\hline \multirow{2}{*}{$\begin{array}{c}\text { Protein } \\
\text { content } \\
(\mathrm{mg} / 100 \mathrm{~g})\end{array}$} & $P$ & -0.0001 & 0.0000 & 0.0010 & 0.0002 & 0.0004 & 0.0005 & -0.0003 & 0.0012 & -0.0003 & 0.0006 & -0.0003 & 0.0009 & 0.0000 & 0.0005 & -0.0011 & -0.0013 & $\underline{-0.0038}$ & 0.0003 & 0.0003 & $-0.1093^{*}$ \\
\hline & G & -0.0113 & -0.0014 & 0.0631 & 0.0151 & 0.0275 & 0.0356 & -0.0201 & 0.0738 & -0.0153 & 0.0334 & -0.0152 & 0.0507 & 0.0020 & 0.0305 & -0.0687 & -0.0767 & $\underline{-0.2237}$ & 0.0214 & 0.0195 & $-0.1095^{*}$ \\
\hline \multirow{2}{*}{$\begin{array}{l}\text { Iodine content } \\
(\mathrm{mg} / 100 \mathrm{~g})\end{array}$} & $P$ & 0.0018 & 0.0021 & 0.0018 & -0.0014 & -0.0016 & -0.0015 & 0.0008 & 0.0023 & -0.0001 & 0.0010 & 0.0027 & 0.0020 & 0.0013 & 0.0021 & 0.0021 & -0.0001 & -0.0004 & $\underline{0.0046}$ & -0.0019 & $0.3896^{*}$ \\
\hline & G & -0.0349 & -0.0384 & -0.0351 & 0.0291 & 0.0306 & 0.0308 & -0.0138 & -0.0431 & 0.0022 & -0.0191 & -0.0492 & -0.0370 & -0.0240 & -0.0398 & -0.0402 & 0.0021 & 0.0081 & $\underline{-0.0846}$ & 0.0355 & $0.3949^{*}$ \\
\hline \multirow{2}{*}{$\begin{array}{c}\text { YVMV } \\
\text { disease } \\
\text { incidence }(\%)\end{array}$} & $P$ & 0.0415 & 0.0442 & 0.0262 & -0.0473 & -0.0516 & -0.0501 & 0.0302 & 0.0264 & 0.0353 & 0.0413 & 0.0480 & 0.0156 & 0.0269 & 0.0469 & 0.0359 & 0.0059 & 0.0068 & 0.0331 & $\underline{-0.0796}$ & -0.5640 \\
\hline & G & 0.4118 & 0.4353 & 0.2607 & -0.5166 & -0.5173 & -0.5317 & 0.3007 & 0.2509 & 0.3352 & 0.3903 & 0.4719 & 0.1486 & 0.2533 & 0.4581 & 0.3391 & 0.0550 & 0.0653 & 0.3134 & $\underline{-0.7470}$ & -0.5652 \\
\hline
\end{tabular}


This observation was in agreement with the results of Reddy et al., (2013) and Yonus et al., (2014).

Days to first flowering showed very high positive indirect effect through fruit weight, number of fruits per plant, number of seeds per fruit, 100 seed weight and total number of pickings also exhibited high positive indirect effect on fruit yield per plant. Number of fruits per plant showed very high positive indirect effect through plant height, number of branches, internodal length, days to last harvest, fruit weight, number of seeds per fruit and number of pickings also exhibited high positive indirect effect on fruit yield per plant (Table 2). This observation was in agreement with the results of Alam and Hossain (2006), Adiger et al., (2011), Das et al., (2012) and Reddy et al., (2013).

The direct effect of all above mentioned traits on fruit yield per plant favours yield improvement through selection. This suggested that indirect selection based on plant height will be effective in yield improvement.

\section{References}

Adeniji, O.T. and Peter, J.M. 2005. Stepwise regression analysis of pod and seed yield characters in segregating F2 population of West African okra (Abelmoschus caillei). Proceedings of 30th Conference, Genetics Society of Nigeria, pp. 250-258.

Adiger, S.G, Shanthkumar, P.I, Gangashetty and Salimath, P.M. 2011. Association studies in okra (Abelmoschus esculentus (L.) Moench). Electron. J. Pl. Breed., 2(4): 568-573.

Alam, A.K.M.A. and Hossain, M.M. 2006. Variability of different yield contributing parameters and yield of some okra (Abelmoschus esculentus (L.)
Moench) accessions. J. Agric. Rural Dev., 4(1\&2): 119-127.

Bendale, V.W, Kadam, S.R, Bhave, S.G, Mehta, J.L. and Pethe, U.B. 2003. Genetic variability and correlation studies in okra. Orissa J. Hort., 31(2): $1-4$.

Camciuc, M, Bessifre, J.M, Vilarem, G. and Gaset, A. 1981. Volatile components in okra seed coat. Phytochem., 48: 311315.

Das, S., Chattopadhyay, A, Chattopadhyay, S.B., Dutta, S and Hazra, P. 2012. Genetic parameters and path analysis of yield and its components in okra at different sowing dates in the Gangetic plains of eastern India. Afr. J. Biotechnol., 11(95): 16132-16141.

Dewey, D.R. and Lu, K.H. 1959. A correlation and path coefficient analysis of components of crusted wheat grass seed production. Agron. J., 51: 515518.

Jaiprakashnarayan, R.P. and Mulge, R. 2004. Correlation and path analysis in okra (Abelmoschus esculentus (L.) Moench). Indian J. Hort., 61(3): 232-235.

Kang, M.S., Miller, J.D. and Tai, P.P. 1983. Genetic and phenotypic path analyses and heritability in sugarcane. Crop Sci., 23: 643-647.

Kochhar, S.L. 1986. Tropical crops. Macmillan Publishers Ltd., London and Basingstoke, pp. 467.

Lenka, D. and Mishra, B. 1973. Path coefficient analysis of yield in rice varieties. Indian J. Agric. Sci., 43: 376379.

Niranjan, R.S. and Mishra, M.N. 2003. Correlation and path coefficient analysis in okra. Prog. Horti., 35: 192-195.

Panse, V.G. and Sukhatme, P.V. 1967. Statistical methods for agricultural workers. ICAR, New Delhi, p. 134-192.

Reddy, T.M., Babu, K.H., Ganesh, M. Reddy, K.C., Begum, H., Reddy, R.S.K. 
and Babu, J.D. 2013. Correlation and path coefficient analysis of quantitative characters in okra (Abelmoschus esculentus (L.) Moench). Songklanakarin. J. Sci. Technol., 35(3): 243-250.

Savello, P., Martin, F.W. and Mill, J.M. 1980. Nutritional composition of okra seed meal. Agri. Food Chem., 28: 11631166.

Simon, S.Y., Gashua, I.B. and Musa, I. 2013. Genetic variability and trait correlation studies in okra (Abelmoschus esculentus (L.). Moench). Agric. Biol. J.N. Am., 4(5): 532-538.

Singh, B., Pal, A.K. and Singh, S. 2006. Genetic variability, correlation and path analysis in okra (Abelmoschus esculentus (L.) Moench). Indian J. Hort., 63(3): 281-285.

Snedecor, G.W. and Cochran, C.W.G. 1967. Statistical methods. The Iowa State University Press, IOWA, U.S.A.

Somashekhar, G., Mohankumar, H.D. and Salimath, P.M. 2011. Genetic analysis of association studies in segregating population of okra. Karnataka J. Agric. Sci., 24(4): 432-435.

Yonus, M, Garedew, W and Debela, A. 2014. Variability and association of quantitative characters among okra (Abelmoschus esculentus (L.) Moench) collection in south western Ethiopia. $J$. Biol. Sci., 14(5): 336-342.

\section{How to cite this article:}

Prasath, G., K. Ravinder Reddy and Pidigam Saidaiah. 2017. Correlation and Path Coefficient Analysis of Fruits Yield and Yield Attributes in Okra [Abelmoschus esculentus (L.) Moench]. Int.J.Curr.Microbiol.App.Sci. 6(3): 462-472. doi: https://doi.org/10.20546/ijcmas.2017.603.054 